\title{
Indicadores de sustentabilidade e influência de sistemas agroflorestal e convencional sobre a qualidade do solo e do café arábica em Piumhi-MG
}

\section{Sustainability indicators and influence of agroforestry and conventional systems on soil quality and arabica coffee in Piumhi-MG}

\author{
Marcos Vinícius Meneses Vieira ${ }^{1}$; Otavio Duarte Giunti ${ }^{2}$ : Cristiane Fortes Gris $^{3}$; Ariana Vieira Silva ${ }^{4}$
}

\begin{abstract}
Resumo: Objetivou-se com esse trabalho a comparação dos teores nutricionais dos solos de uma área constituída por um SAF (Sistema Agroflorestal), que tem o cafeeiro como um dos seus principais elementos constituinte, com os de uma área com o café em monocultivo convencional e a confrontação dos teores nutricionais do solo do SAF antes e três anos após sua implantação, bem como a seleção e aplicação de indicadores de sustentabilidade para avaliar e contrastar parâmetros relacionados à qualidade do solo e do cafeeiro nos dois sistemas avaliados. Na comparação entre análises de solo de amostras retiradas da linha e entrelinha do café convencional e do SAF, os valores de $\mathrm{pH}, \mathrm{P}, \mathrm{K}, \mathrm{Ca}, \mathrm{Mg}$ e MO apresentaram-se mais elevados nas amostras de solo retiradas na entrelinha do cafeeiro convencional e na linha de plantio do SAF, na profundidade de 0-20 cm. . Nas comparações entre análise da área total do SAF retirada em período anterior à sua implantação com análises atuais das linhas e entrelinhas do SAF, os teores de P, K e MO na linha de plantio mostraram-se 302\%, 144,8\% e 177\% superiores, respectivamente, aos dos obtidos antes da implantação do SAF, enquanto que nas entrelinhas, esses teores mostraram-se $165 \%, 135,8 \%$ e 151,5\% superiores. Os teores de Ca e Mg apresentaram uma leve redução, com exceção dos teores de $\mathrm{Mg}$ na linha, que apresentou elevação de $13 \%$. Em relação à aplicação dos indicadores de sustentabilidade, o SAF apresentou-se melhor quanto aos indicadores de qualidade do solo. Entretanto, quando comparado com o monocultivo, os critérios agronômicos de produtividade para o SAF mostraram-se baixos.
\end{abstract}

Palavras-chave: Monocultura, agroecossistemas, $C$. arabica L, matéria orgânica, nutrientes.

Abstract: The objective of this work to compare the nutritional content of the soil of an area consisting of a AFS ( Agroforestry System), which has the coffee as one of its main constituent elements, with an area with coffee in conventional monoculture and the comparison of the AFS soil nutrient levels before and three years after its implementation, as well as the selection and application of sustainability indicators to assess and contrast parameters related to soil quality and the coffee in both systems evaluated. Comparing soil analysis of samples taken from row and between row of conventional coffee and $\mathrm{SAF}$, $\mathrm{pH}$ values, $\mathrm{P}, \mathrm{K}, \mathrm{Ca}, \mathrm{Mg}$ and $\mathrm{MO}$ were more elevated in soil samples taken in between row of conventional coffee and in the AFS plant line at a depth of 0-20 cm. In the comparisons between analysis of the total area of the AFS withdrawn in the period prior to its implementation with current analyzes of rows and between rows of AFS, the levels of P, K and MO in the row proved to be $302 \%, 144.8 \%$ and $177 \%$ higher, respectively, to those obtained before the implementation of the AFS, while between rows, these levels proved to be $165 \%, 135.8 \%$ and $151.5 \%$ higher. Ca and $\mathrm{Mg}$ showed a slight decrease, except for Mg on the row, which increased by $13 \%$. When the application of sustainability indicators, the AFS presented better in relation to soil quality indicators. However, when compared with conventional tillage cultivation in monoculture, agronomic productivity criteria for AFS shown to be low.

Key words: Monoculture, agroecosystems, C. arabica L, organic matter, nutrients.

\footnotetext{
*Autor para correspondência

Recebido para publicação em 16/03/2015; aprovado em 16/05/2015

${ }^{1}$ Pós-graduando em Cafeicultura, Instituto Federal de Educação, Ciência e Tecnologia do Sul de Minas Gerais - Campus Muzambinho. Muzambinho - MG. E-mail: marcos_vm_vieira@yahoo.com.br

${ }^{2}$ Professor Especialista, Instituto Federal de Educação, Ciência e Tecnologia do Sul de Minas Gerais - Campus Muzambinho. Muzambinho - MG. E-mail: otavio.ifsuldeminas@gmail.com

${ }^{3}$ Professora Doutora, Instituto Federal de Educação, Ciência e Tecnologia do Sul de Minas Gerais - Campus Muzambinho. Muzambinho - MG. E-mail: cristiane.gris@muz.ifsuldeminas.edu.br

${ }^{4}$ Professora Doutora, Instituto Federal de Educação, Ciência e Tecnologia do Sul de Minas Gerais - Campus Muzambinho. Muzambinho - MG. E-mail: ariana.silva@muz.ifsuldeminas.edu.br
} 


\section{INTRODUÇÃO}

O cultivo de cafezais em sistemas agroflorestais (SAF's) é uma alternativa na construção de um sistema de produção sustentável para agricultores familiares residentes nas áreas tradicionalmente produtoras de café. Os sistemas agroflorestais são formas de manejo em cultivo múltiplo, na qual espécies de cultivo anual ou perenes são utilizadas em associação com pelo menos uma espécie arbórea (SOMMARIBA, 1992).

Os SAF's têm como objetivo otimizar os efeitos benéficos das interações existentes no ecossistema, a fim de proporcionar maior diversidade de produtos, reduzir a necessidade de insumos externos e os impactos negativos das explorações agrícolas sobre o meio ambiente e aumentar a resiliência e a capacidade adaptativa dos agroecossistemas (GLIESSMAN, 2005).

O aumento ou formação de uma camada de serrapilheira em cultivos de culturas perenes é um fator importante à manutenção da matéria orgânica do solo. Nos ambientes tropicais, o processo de degradação dos solos está intimamente relacionado com a dinâmica da matéria orgânica. Assim, conversão da vegetação nativa em áreas de produção agrícola pode reduzir drasticamente os teores de matéria orgânica do solo, devido ao menor suprimento de resíduos e o aumento da taxa de decomposição, bem como a elevação nas perdas das camadas superficiais do solo por erosão (ROSCOE et al., 2006).

Uma das etapas mais importantes de ciclagem de nutrientes é a decomposição da camada de serrapilheira depositada sobre o solo. O acúmulo de serrapilheira no solo é determinado pela quantidade de material deposto sobre o mesmo e a velocidade de decomposição desta camada. Quantidades significativas de nutrientes podem retornar ao solo através da queda de componentes senescentes de plantas, sendo determinantes na sustentabilidade de ecossistemas naturais estabelecidos em solos de baixa fertilidade (CORREIA; ANDRADE, 1999).

$\mathrm{O}$ manejo em sistemas agroflorestais baseados em processos ecológicos busca a recuperação da camada de serrapilheira sobre o solo para melhorar o fluxo de energia e ciclagem de nutrientes. Campanha et al. (2007) registraram o maior acúmulo de serrapilheira em café conduzido em sistema agroflorestal quando comparado com café em sistema convencional na região de Viçosa - MG, fator que contribuiu para que o SAF apresentasse maior teor médio de umidade no solo na camada de $20-40 \mathrm{~cm}$ do solo, melhorando também a saturação por bases, reduzindo o teor de alumínio trocável e aumentando a CTC do solo.

Com o aporte continuo de resíduos vegetais no solo, a produção de ácidos orgânicos de baixa massa molecular, que promovem efeitos na melhoria das características químicas do solo é mantida. Em sistemas de aporte contínuo, Pavinato e Rosolem (2008) observaram a elevação do pH do solo devido à complexação de $\mathrm{H}$ e $\mathrm{Al}$ livres com os compostos orgânicos aniônicos e o aumento da saturação da CTC por $\mathrm{Ca}, \mathrm{Mg}$ e $\mathrm{K}$ adicionados via resíduo vegetal.

A introdução de árvores no sistema de produção altera o ambiente luminoso pelo sombreamento o qual, por sua vez, afeta a umidade, evapotranspiração e temperatura (GLIESSMAN, 2005). Villatoro (2004) destaca que o sombreamento é um dos mais importantes efeitos da arborização, por afetar diretamente a interceptação da radiação solar e a eficiência fotossintética e respiratória dos cafeeiros. Os benefícios da sombra nos cafeeiros mostram-se mais evidentes em situações de limitações ambientais, como as que se relacionam à fertilidade do solo, temperaturas extremas e estações secas bem definidas.

A máxima taxa fotossintética em cafeeiros é atingida em condições de irradiância moderada (KUMAR; TIESZEN, 1980). Devido a fatores como fotoinibição e danos ao fotossistema II, a eficiência fotossintética é reduzida em condições de elevada irradiância, promovendo um incremento da temperatura foliar, com consequências na redução na condutância do mesófilo, o que contribui para prejudicar as trocas gasosas na folha (RAMALHO et al., 1998; NUNES et al., 2003; FREITAS et al., 2003). Desse modo, segundo Villatoro (2004), benefícios relacionados às modificações do microclima causadas pela cobertura arbórea, principalmente no que diz respeito ao controle da irradiação e flutuações extremas de temperatura, são esperados em associações entre o cafeeiro e espécies arbóreas.

Para auxiliar na transição de agroecossistemas, uma boa ferramenta é o uso de indicadores de fácil aplicação, que permite aos agricultores familiares caracterizar e monitorar seus sistemas, avaliar e tomar decisões, definir ou monitorar a sustentabilidade de sua realidade, determinar os pontos críticos e buscar novas tecnologias que promovam melhorias na qualidade de seu agroecossistema (ALTIERI; NICHOLS, 2002).

Verona et al. (2007) definem um indicador como uma medida do comportamento de um sistema em termos de condições expressivas e perceptíveis. Moura et al. (2004) caracterizam indicador de sustentabilidade como um "conjunto de parâmetros que permitam medir as modificações antrópicas em um determinado sistema e comunicar, de forma simplificada, o estado deste sistema em relação aos critérios e as metas estabelecidas para avaliar a sua sustentabilidade".

Diante do exposto, objetivou-se com este trabalho a comparação dos teores nutricionais dos solos de uma área constituída por um SAF, que tem o cafeeiro como um dos seus principais elementos constituinte com os de uma área com o café em monocultivo convencional, assim como a comparação dos teores nutricionais do solo do SAF antes e três anos após sua implantação, buscando avaliar a influência do sistema sobre características químicas do solo e a seleção e aplicação de indicadores de sustentabilidade para avaliar e comparar parâmetros relacionados à qualidade do solo e do cafeeiro nos dois sistemas avaliados.

\section{MATERIAL E MÉTODOS}

O estudo foi realizado na Fazenda Água Limpa, no município de Piumhi, oeste do estado de Minas Gerais, que apresenta clima regional classificado como Cwa, segundo Köppen (1948), que indica clima temperado úmido, com inverno seco e verão quente.

Estudaram-se duas áreas, uma de 0,5 hectares de SAF e outra de café convencional, considerando-se um talhão de três hectares mais próximo à área do SAF.

O SAF foi implantado em novembro de 2011 em uma área usada há 10 anos com cultivo de milho e feijão, e 
encontrava-se em pousio há um ano. Na implantação do SAF foi feito apenas o sulco de plantio e adicionado esterco de gado ao sulco, na quantidade de 5 litros por metro linear. Foram plantadas 1000 mudas de café da cultivar Catuaí Vermelho, no espaçamento de 3,00 m x 0,80 m, associado a mudas de espécies arbóreas na linha de plantio.

Dentre as espécies arbóreas, plantadas de forma intercalada e com respectivos espaçamentos encontravam-se o Pau de Balsa (Ochoroma pyramidale) - 3 m x 6 m, Acácia Australiana (Acacia mangium) - $3 \mathrm{~m}$ × $6 \mathrm{~m}$, Gliricídia (Gliricidia sepium Jacq. Steud) $-3 \mathrm{~m}$ x $3 \mathrm{~m}$ e bananeira
(Musa spp.) - $9 \mathrm{~m}$ × $6 \mathrm{~m}$. Foram ainda inseridas outras espécies sem espaçamento definido, tais como Goiaba (Psidiun guajava), Canafistula (Pelthoforum dubium), Angico vermelho (Anadenanthera macrocarpa), Paineira (Ceiba speciosa A. St.-Hil.), Tamarindo (Tamarindus indica L.), Ipê (Tabebuia serratifolia) e Jacarandá mimoso (Jacaranda mimosaefolia). Nas linhas de plantio, foram inseridas também a mucuna preta (Mucuna aterrima), o margaridão (Tithonia diversifolia) e o feijão guandu anão (Cajanus cajan L.). A Figura 1 apresenta um croqui com as espécies plantadas na linha do SAF.

Figura 1: Croqui da implantação do SAF, espécies, espaçamento e distribuição das mudas nas linhas de plantio.



\begin{tabular}{|c|c|c|c|}
\hline Legenda: & \& Gliricídea & Goiaba & Banana \\
\hline (8) Café & Pau de Balsa & Acácia Mangium & Tै Espécies Diversas \\
\hline
\end{tabular}

Fonte: Autores (2014).

Para a geração de biomassa a ser podada foram plantados, nas entrelinhas, Crotalaria juncea, Crotalaria spectabilis, feijão-de-porco (Canavalia ensiformis) e milho (Zea mays). O plantio dessas espécies foi feito em cinco linhas, sendo a linha central ocupada pelo feijão-de-porco, as duas linhas laterais constituídas pelo milho e as duas linhas de bordadura compostas pelas crotalárias.

Após a implantação do SAF, anualmente foi feito o manejo de poda do margaridão e também o raleamento das touceiras de bananeira, procurando-se deixar uma ou duas bananeiras por touceira, com o material vegetal resultante destes manejos colocado ao lado da linha de café.

Com um ano e meio de implantação do SAF, as plantas de feijão guandu foram cortadas e os resíduos vegetais depositados nas linhas de café. Por essa ocasião, algumas plantas de Pé de Balsa, derrubadas pelo vento, também foram cortadas e depositadas ao lado das linhas de plantio de café. Após um período de aproximadamente 150 dias após o plantio da mucuna preta, esta foi cortada, com os resíduos vegetais depositados ao lado das linhas de café.

Nos anos de 2013 e 2014 as plantas de gliricidía foram podadas na altura de $1,5 \mathrm{~m}$, retirando-se cerca de $30 \%$ a $50 \%$ da copa das árvores. Em 2013, as plantas de Acácia tiveram os ramos inferiores podados, incentivando seu crescimento, deixando-se as plantas com fuste único e, em 2014, foi realizada uma poda de sua copa, também se retirando entre
$30 \%$ a $50 \%$ da mesma $\mathrm{O}$ material vegetal resultante dessas podas foi depositado no solo, ao lado das linhas de café.

Após a colheita dos grãos de milho, que ocorreu aproximadamente 160 dias após a semeadura, as crotalárias e o feijão-de-porco foram roçados. Houve germinação espontânea de mamona (Ricinus communis L.) do banco de sementes do solo, realizando-se um manejo para raleamento dessa espécie, deixando-se alguns indivíduos para favorecer um sombreamento inicial. Após a roçada dos adubos verdes, foi plantado, na entrelinha, capim colonião (Panicum maximum Jacq CV Colonião). Em 2012, o capim colonião foi roçado trimestralmente e, em 2013 e 2014, foi realizada apenas uma roçada anual.

$\mathrm{Na}$ área de café convencional, implantada há 25 anos, a correção do solo é feita a cada três anos, sendo a última calagem realizada em outubro de 2011, aplicando-se $2.000 \mathrm{~kg}$ $\mathrm{ha}^{-1}$ de calcário dolomítico. Anualmente, é realizada uma adubação de produção, utilizando-se o formulado comercial 20-05-20. O manejo do mato nos últimos quatro anos foi realizado por meio de roçadas.

Para o estudo de caso, em agosto de 2014, foram feitas amostragens de solo nos dois sistemas (SAF e Café convencional) nas profundidades de $0-20 \mathrm{~cm}$ e $20-40 \mathrm{~cm}$, na linha e na entrelinha. Cada amostra foi composta de 10 subamostras, coletadas em 10 pontos de cada área, com caminhamento em zigue-zague pelo local. As amostras foram 
encaminhadas para o Laboratório de Solos e Folhas do IFSULDEMINAS, Campus Muzambinho, para que fossem analisados os teores de macro e micronutrientes, matéria orgânica, pH e teor de alumínio. Utilizou-se também de uma análise de solo realizada no SAF em 2011, com amostragem feita na profundidade de 0-20 $\mathrm{cm}$, antes da implantação do sistema, para efeitos de comparação com os resultados atuais.

Para a avaliação da sustentabilidade dos sistemas foi utilizada a metodologia de indicadores de rápida aplicação, proposta por Altieri e Nichols (2002). A aplicação da metodologia foi realizada por dois Engenheiros Agrônomos com conhecimento e experiência de campo em cultura de café, com as avaliações e as notas dadas em conjunto por esses dois avaliadores. Assim, para esse estudo, foram

\section{RESULTADOS E DISCUSSÃO}

A Tabela 1 apresenta os resultados das análises de solo nos dois sistemas analisados (SAF e café realizadas as seguintes etapas, adaptadas de Deponti et al. (2002): escolha da área a ser analisada, definição dos atributos ou características de sustentabilidade, definição dos descritores, levantamento dos indicadores adequados, seleção dos indicadores estratégicos, determinação dos parâmetros, aplicação dos indicadores no local de estudo e mensuração e análises dos resultados.

Com base nessas informações, foram selecionados, adaptados e aplicados indicadores que se relacionam à qualidade do solo (quatorze indicadores) e do cafeeiro (sete indicadores), para os dois sistemas analisados. Para cada indicador foram atribuídas as notas 1, 5 ou 10, de acordo com as características avaliadas.

convencional) nas profundidades de $0-20 \mathrm{~cm}$ e $20-40 \mathrm{~cm}$, na linha e entrelinha de plantio do café.

Tabela 1 - Resultados analíticos do solo de um SAF e cafeeiro convencional, nas profundidades de 0-20 e 20-40 cm. Fazenda Água Limpa, Piumhi, MG, 2014.

\begin{tabular}{|c|c|c|c|c|c|c|c|c|c|c|}
\hline \multirow[t]{2}{*}{ Parâmetro } & \multirow[t]{2}{*}{ Extrator } & \multirow[t]{2}{*}{ Unidade } & \multicolumn{2}{|c|}{ Café linha } & \multicolumn{2}{|c|}{ Café entrelinha } & \multicolumn{2}{|c|}{ S AF linha } & \multicolumn{2}{|c|}{ S AF entrelinha } \\
\hline & & & $0-20 \mathrm{~cm}$ & $20-40 \mathrm{~cm}$ & $0-20 \mathrm{~cm}$ & $20-40 \mathrm{~cm}$ & $0-20 \mathrm{~cm}$ & $20-40 \mathrm{~cm}$ & $0-20 \mathrm{~cm}$ & $20-40 \mathrm{~cm}$ \\
\hline $\mathrm{pH}$ & $\left(\mathrm{H}_{2} \mathrm{O}\right)$ & & 4,97 & 4,83 & 6,86 & 7,2 & 6,77 & 6,82 & 6,65 & 5,58 \\
\hline $\mathrm{P}$ & $\left(\right.$ Mehlich $\left.^{-1}\right)$ & $\mathrm{mg} / \mathrm{dm}^{3}$ & 9 & 2,4 & 38,9 & 8,2 & 11,5 & 2,4 & 6,3 & 10,6 \\
\hline $\mathrm{K}$ & $\left(\right.$ Mehlich $\left.^{-1}\right)$ & $\mathrm{mg} / \mathrm{dm}^{3}$ & 340 & 239 & 530 & 300 & 565 & 455 & 530 & 202 \\
\hline $\mathrm{Ca}$ & $\left(\mathrm{KCl}^{-1} \mathrm{~mol} / \mathrm{L}\right)$ & $\mathrm{cmol}_{\mathrm{d}} / \mathrm{dm}^{3}$ & 1,24 & 0,66 & 6,44 & 3,54 & 4,01 & 3,36 & 3,49 & 2,25 \\
\hline $\mathrm{Mg}$ & $\left(\mathrm{KCl}^{-1} \mathrm{~mol} / \mathrm{L}\right)$ & $\mathrm{cmol}_{d} / \mathrm{dm}^{3}$ & 0,42 & 0,23 & 2,55 & 1,41 & 1,46 & 1,16 & 1,08 & 0,7 \\
\hline $\mathrm{Al}$ & $\left(\mathrm{KCl}^{-1} \mathrm{~mol} / \mathrm{L}\right)$ & $\mathrm{cmol}_{d} / \mathrm{dm}^{3}$ & 1,44 & 1,82 & 0 & 0 & 0 & 0 & 0 & 0,13 \\
\hline $\mathrm{H}+\mathrm{Al}$ & (SMP) & $\mathrm{cmol}_{d} / \mathrm{dm}^{3}$ & 7,83 & 8,34 & 1,64 & 1,92 & 2,13 & 2,04 & 2,37 & 4,17 \\
\hline M.O. & (Oxi-Red.) & $\mathrm{dag} / \mathrm{kg}^{3}$ & 3,41 & 2,87 & 4,76 & 2,74 & 3,99 & 2,74 & 3,41 & 3,28 \\
\hline $\mathrm{Zn}$ & $\left(\right.$ Mehlich $\left.^{-1}\right)$ & $\mathrm{mg} / \mathrm{dm}^{3}$ & 2,8 & 0,9 & 10,6 & 2,5 & 11,5 & 5,9 & 9,6 & 7,5 \\
\hline $\mathrm{Fe}$ & $\left(\right.$ Mehlich $\left.^{-1}\right)$ & $\mathrm{mg} / \mathrm{dm}^{3}$ & 22,2 & 21,2 & 41,8 & 31,5 & 56,5 & 49,7 & 51,4 & 60,8 \\
\hline $\mathrm{Mn}$ & $\left(\right.$ Mehlich $\left.^{-1}\right)$ & $\mathrm{mg} / \mathrm{dm}^{3}$ & 15 & 8,1 & 82,9 & 24,4 & 33,6 & 20,8 & 26,8 & 22,8 \\
\hline $\mathrm{Cu}$ & $\left(\right.$ Mehlich $\left.^{-1}\right)$ & $\mathrm{mg} / \mathrm{dm}^{3}$ & 3,9 & 2,2 & 4,8 & 3,1 & 3,5 & 3,5 & 5,4 & 3,8 \\
\hline B & (Água quente) & $\mathrm{mg} / \mathrm{dm}^{3}$ & 0,46 & 0,31 & 0,37 & 0,2 & 0,28 & 0,22 & 0,16 & 0,28 \\
\hline$S$ & (Fosfato Monoc.) & $\mathrm{mg} / \mathrm{dm}^{3}$ & 50,6 & 123,4 & 7,6 & 14,6 & 5,7 & 6,6 & 4,2 & 9 \\
\hline P-rem. & $\left(\mathrm{CaCl}_{2}\right)$ & $\mathrm{mg} / \mathrm{L}$ & 9,4 & 4,3 & 14,4 & 8,6 & 14,4 & 8 & 12,2 & 21,9 \\
\hline S.B. & (Soma de bases) & $\mathrm{cmol}_{d} / \mathrm{dm}^{3}$ & 2,5 & 1,5 & 10,4 & 5,7 & 6,9 & 5,7 & 5,9 & 3,5 \\
\hline $\mathrm{t}$ & (CTC efetiva) & $\mathrm{cmol}_{d} / \mathrm{dm}^{3}$ & 4 & 3,3 & 10,4 & 5,7 & 6,9 & 5,7 & 5.9 & 3,6 \\
\hline $\mathrm{T}$ & (CTC) & $\mathrm{cmol}_{d} / \mathrm{dm}^{3}$ & 10,4 & 9,8 & 12 & 7,6 & 9,1 & 7,7 & 8,3 & 7,6 \\
\hline $\mathrm{V}$ & (Sat. Bases) & \# & 24,2 & 15,2 & 86,3 & 74,9 & 76,5 & 73,6 & 71,4 & 45,4 \\
\hline $\mathrm{m}$ & (Sat. Al) & $\%$ & 36,3 & 54,8 & 0 & 0 & 0 & 0 & 0 & 3,6 \\
\hline
\end{tabular}

*Análises realizados no Laboratório de Solos e Folhas do IFSULDEMINAS - Campus Muzambinho.

De acordo com as análises de solo, os valores de $\mathrm{pH}, \mathrm{P}$, $\mathrm{K}, \mathrm{Ca}, \mathrm{Mg}$ e MO apresentaram-se mais elevados nas amostras de solo retiradas na entrelinha do cafeeiro convencional e na linha de plantio do SAF, na profundidade de $0-20 \mathrm{~cm}$.

$\mathrm{O}$ pH apresentou valores mais baixos na linha do cafeeiro convencional, indicando solo ácido. Tal resultado aponta que a realização da calagem a cada três anos não é suficiente para reduzir a acidificação do solo, que também pode ser intensificada pelas adubações químicas anuais, conforme salientou Primavesi (2002).

Em relação aos teores de $\mathrm{P}$, em função do P-rem (Fósforo remanescente), para a profundidade de 0-20 cm, segundo Guimarães et al. (1999), estes encontram-se na classe de fertilidade bom para a linha de plantio do café 
convencional e para o SAF na entrelinha e muito bom para a entrelinha de café convencional e SAF na linha. Quanto aos teores de $\mathrm{K}$, em todos os locais amostrados, o teor desse nutriente encontra-se em níveis elevados. Os teores de $\mathrm{Ca}$ e $\mathrm{Mg}$ mostraram-se baixos na linha do cafeeiro convencional, que podem ser consequência do $\mathrm{pH}$ baixo, que indisponibiliza esses nutrientes (RAIJ et al., 1997). Também podem ser explicados pela arruação realizada na lavoura, que retirou os resíduos vegetais embaixo dos pés de café. Além de esses resíduos servirem como fontes de nutrientes, a arruação sem a posterior esparramação dos resíduos, que é uma prática comum na propriedade, por deixar o solo exposto, pode intensificar os processos erosivos do solo, reduzindo a fertilidade dos mesmos.

Já em relação a MO, teores considerados altos por Matiello; Garcia; Almeida (2006) foram observados em todos os locais, para ambas as profundidades. Mesmo apresentando teores elevados nos dois sistemas, nos locais e profundidades amostradas, a matéria orgânica apresentou-se superior na entrelinha do café convencional e na linha de plantio do SAF. Para os dois sistemas, estas são as regiões de maior acúmulo de matéria orgânica, pois no SAF, além do manejo do margaridão, feijão guandú e mucuna e poda das árvores na linha de plantio, o capim roçado na entrelinha é rastelado para a linha de plantio, formando-se assim um camalhão de cobertura morta. Já no cafezal convencional, o maior acúmulo de matéria orgânica entre as linhas de plantio se deve ao fato da realização da arruação debaixo da saia do café e, este material retirado não ser realocado para debaixo da saia. $\mathrm{O}$ fato de o café covencional ter apresentado maior teor de matéria orgânica que o SAF pode ser explicado pelo manejo do SAF não ter contemplado as podas e roçadas em número suficiente para fornecer uma biomassa adequada ao sistema. Outro fator que pode ter influenciado é o fato do SAF provavelmente apresentar uma condição climática mais amena, com redução da amplitude térmica e elevação da umidade dentro da lavoura, favorecendo a atividade microbiana e resultando numa aceleração do processo de decomposição no SAF, conforme observado por Campanha et al.(2007).

$\mathrm{O} \mathrm{N}$ e o K são os nutrientes mais exigidos por cafeeiros, principalmente na fase adulta, com maior necessidade de $\mathrm{N}$ para a vegetação e de $\mathrm{K}$ para a produção, com ligeira superioridade de N (20\%) no cômputo global (MATIELLO et al., 2002).

Teores considerados altos de $\mathrm{S}$ foram encontrados nas amostras retiradas na linha do cafeeiro convencional, nas duas profundidades amostradas e na profundidade de $20-40 \mathrm{~cm}$, na entrelinha do cafeeiro convencional. Nas demais situações, com exceção da entrelinha do SAF na profundidade de 0-20 $\mathrm{cm}$, cujos teores mostraram-se baixos, os níveis verificados para esse nutriente encontram-se médios (MATIELLO;
GARCIA; ALMEIDA, 2006,). Na linha do café convencional, os teores desse nutriente mostraram-se extremamente elevados; em comparação com a linha de plantio do SAF, os valores apresentaram-se $888 \%$ superiores, na profundidade de $0-20 \mathrm{~cm}$. Uma explicação pode ser o fato que, no cafeeiro convencional, anualmente é realizada adubação com o formulado comercial 20-05-20, que pode ter em sua composição o S, oriundo do Sulfato de Amônio que pode ser utilizado em sua formulação.

Em relação aos micronutrientes, na profundidade de 0 $20 \mathrm{~cm}$, os teores de $\mathrm{Fe}$ e $\mathrm{Cu}$ encontram-se elevados nos dois sistemas avaliados; em relação aos teores de $\mathrm{Zn}$ e $\mathrm{Mg}$, estes mostram-se elevados, com exceção da linha do cafeeiro convencional, cujos teores encontram-se médios. Já os teores de B situam-se baixos nos dois sistemas

Em situações de teor alto de nutrientes, são necessárias adubações de manutenção da cultura, podendo-se reduzir ou até mesmo dispensar o fornecimento de nutrientes cujos níveis mostrarem-se suficientes (MATIELLO; GARCIA; ALMEIDA, 2006).

Para a linha do cafezal o valor de saturação por bases (V\%) está em $24,4 \%$, considerado muito baixo para a cultura do café, enquanto que para a entrelinha do cafezal e a linha de plantio do SAF este valor está em 86,3\% e 76,5\%, respectivamente, indicando uma quantidade maior de cátions disponíveis na solução do solo. De acordo com Matiello, Garcia e Almeida (2006), valores de V\% abaixo de 40 são considerados baixos para a cultura do café, enquanto valores acima de $60 \%$ são considerados elevados. Os baixos teores de $\mathrm{Ca}$ e $\mathrm{Mg}$ na linha de plantio, associados a teores elevados de $\mathrm{H}+\mathrm{Al}$ influenciam na saturação de bases. A saturação por $\mathrm{Al}$ na linha de plantio do café convencional está em 36,3\% enquanto que para a sua entrelinha e a linha de plantio do SAF esta se encontra em $0 \%$. Teores elevados de $\mathrm{Al}$ comprometem a absorção de $\mathrm{Ca}$ pelas plantas, podendo resultar em menor crescimento do sistema radicular (CARVALHO, 2008). A calagem insuficiente, associada ao manejo de arruação após a colheita podem ter influenciado os teores de $\mathrm{Ca}$ e $\mathrm{Mg}$, resultando em teores mais elevados de saturação por alumínio.

Estas diferenças observadas na análise de solo indicam o grande poder de estruturação físico química da matéria orgânica nestes solos e que a falta de um manejo que promova o acúmulo de matéria orgânica se torna insustentável, necessitando constantemente de novas correções de solo para neutralizar o poder tóxico do alumínio e recompor a saturação por bases mínimas demandadas pelo café.

A Tabela 2 apresenta um comparativo da análise de solo realizada em área total no SAF em 2011, ano de sua implantação, e em agosto de 2014, na linha e entrelinha na profundidade de $0-20 \mathrm{~cm}$.

Tabela 2 - Resultados Analíticos temporais do solo do SAF, na profundidade de 0-20 cm. Fazenda Água Limpa, Piumhi, MG.

\begin{tabular}{|c|c|c|c|c|c|c|c|c|c|c|c|c|c|c|}
\hline \multirow[t]{2}{*}{ Amostras } & \multirow[t]{2}{*}{ Ano } & \multirow[t]{2}{*}{$\mathrm{pH}$} & \multirow{2}{*}{$\frac{P}{\mathrm{mg} / \mathrm{dm}^{3}}$} & \multirow{2}{*}{$\frac{\mathrm{K}}{\mathrm{mg} / \mathrm{dm}^{3}}$} & \multirow{2}{*}{$\frac{\mathrm{Ca}}{\mathrm{cmol}_{\mathrm{d}} / \mathrm{dm}^{3}}$} & \multirow{2}{*}{$\frac{\mathrm{Mg}}{\mathrm{cmol}_{\mathrm{d}} / \mathrm{dm}^{3}}$} & \multirow{2}{*}{$\frac{\mathrm{Al}}{\mathrm{cmol}_{\mathrm{c}} / \mathrm{dm}^{3} \mathrm{c}}$} & \multirow{2}{*}{$\mathrm{H}+\mathrm{Al}$} & \multirow{2}{*}{$\mathrm{SB}$} & \multirow{2}{*}{$\frac{(\mathrm{t})}{\mathrm{mol}_{\mathrm{c}} / \mathrm{dm}^{3}}$} & \multirow{2}{*}{$\frac{(\mathrm{T})}{\mathrm{mol}_{\mathrm{c}} / \mathrm{dm}^{3}}$} & \multirow{2}{*}{$\mathrm{V}$} & \multirow{2}{*}{$\frac{\mathrm{m}}{\%}$} & \multirow{2}{*}{$\frac{\text { M.O. }}{\mathrm{dag}^{\mathrm{kgg}}{ }^{3}}$} \\
\hline & & & & & & & & & & & & & & \\
\hline Área total & 2011 & 6,1 & 3,8 & 390 & 4,24 & 1,29 & 0 & 3,17 & 6,53 & 6,53 & 9,7 & 67,32 & 0 & 2,25 \\
\hline Linha & 2014 & 6,77 & 11.5 & 565 & 4,01 & 1,46 & 0 & 2,13 & 6,9 & 6,9 & 9,1 & 76,5 & 0 & 3,99 \\
\hline Entrelinha & 2014 & 6,65 & 6,3 & 530 & 3,49 & 1,08 & 0 & 2,37 & 5,9 & 5,9 & 8.3 & 71,4 & 0 & 3,41 \\
\hline
\end{tabular}


Conforme os dados da Tabela 2, o efeito da ciclagem de nutrientes no solo fica evidente quando comparadas as análises feitas em 2011 e 2014 para a faixa de $0-20 \mathrm{~cm}$. Os valores de $\mathrm{pH}, \mathrm{P}, \mathrm{K}, \mathrm{MO}$ e V\% mostram-se mais elevados nas análises realizadas em 2014, tanto na linha, quanto na entrelinha do SAF, quando comparados ao ano de sua implantação.

Com o aporte continuo de resíduos vegetais no solo a produção de ácidos orgânicos de baixa massa molecular é mantida. Esses ácidos têm efeitos na melhoria das características químicas do solo. Em sistemas de aporte contínuo, foi possível observar a elevação do $\mathrm{pH}$ do solo devido à complexação de $\mathrm{H}$ e $\mathrm{Al}$ livres com os compostos orgânicos aniônicos, o aumento da saturação da CTC (Capacidade de Troca Catiônica) por $\mathrm{Ca}^{2^{+}}, \mathrm{Mg}^{2+}$ e $\mathrm{K}^{+}$ adicionados via resíduo vegetal (PAVINATO; ROSOLEM, 2008).

Através de seu sistema radicular, espécies arbóreas promovem a absorção e reciclagem de elementos químicos nutricionais presentes no solo e, através da queda de suas folhas, frutos, galhos e troncos realizam a liberação desses nutrientes, podendo apresentar efeito significativo nos níveis de matéria orgânica do solo, que possui grande importância para a manutenção da fertilidade dos solos (MOREIRA, 2009). A matéria orgânica, em conjunto com os óxidos e sesquióxidos existentes no solo, forma os agregados, que cimentam as partículas de areia, silte e argila, originando pequenos agregados, que contribuem para a estruturação dos solos (KIEHL, 2008). Além disso, a matéria orgânica condiciona características químicas do solo, por ser fonte de nutrientes às plantas, sendo que em solos tropicais e subtropicais, a CTC da matéria orgânica pode representar um percentual elevado da CTC total do solo e características biológicas do solo, por atuar como fonte de carbono e energia aos microrganismos quimioheterotróficos e, devido à mineralização de $\mathrm{N}$ e $\mathrm{S}$ orgânico, fornece energia aos microrganismos quimioautotróficos, que possuem grande importância nos solos (BAYER; MIELNICZUK, 2008).

Os teores de $\mathrm{P}, \mathrm{K}$ e $\mathrm{MO}$ na linha de plantio mostraramse $302 \%, 144,8 \%$ e $177 \%$ superiores, respectivamente, aos obtidos em área total, antes da implantação do SAF, enquanto que nas entrelinhas, esses teores mostraram-se 165\%, 135,8\% e 151,5\%, respectivamente superiores à época de implantação.

Avaliando a deposição de resíduos orgânicos no solo em SAF, quando comparados à monocultura, Perez Marin (2002) verificou que no SAF essa deposição mostrou-se até $95 \%$ superior ao solo com convencional, o que permitiu incrementos de nutrientes de até $82 \%$ de N, $119 \%$ de P, $175 \%$ de $\mathrm{K}, 6 \%$ de $\mathrm{Ca}, 34 \%$ de $\mathrm{Mg}$ e $75 \%$ de $\mathrm{S}$, demonstrando a potencialidade no fornecimento de nutrição ao cafeeiro pelos resíduos arbóreos.
De acordo com Matiello, Garcia e Almeida (2006), os teores de MO, que antes da implantação do SAF encontravase em níveis médios, situam-se, atualmente, em níveis elevados; já os teores de $\mathrm{P}$ na linha passaram de baixos para médios.

A maior disponibilidade de fósforo no solo pode ser creditada ao poder de solubilização deste nutriente pelos ácidos orgânicos, como observado por Campanha et al. (2007) e Pavinato e Rosolem (2008). Assim, essa elevação na disponibilidade pode ser devido à inibição da adsorção de $\mathrm{P}$ pela competição com os ácidos orgânicos, que ocupam os sítios de adsorção, resultando em seu melhor aproveitado pelas plantas. Outros autores creditam essa elevação da disponibilidade de $\mathrm{P}$ a uma maior mineralização desse nutriente pela adição de material orgânico aos solos (PAVINATO; ROSOLEM, 2008). Além disso, alguns estudos indicam que o margaridão (Tithonia diversifolia) possui uma maior capacidade de acumulo de $\mathrm{P}$ em seus tecidos (NG'INJA et al.,1998; ARAGÃO et al., 2012), apresentando altas concentrações de $\mathrm{P}$ na fitomassa, entre 0,3 e $0,38 \%$ de $P$, mais elevado que nas demais plantas utilizadas em SAF, conforme verificado por Devide (2013) e Souza Júnior (2007). Pypers et al. (2005) observaram aumento gradual nos teores de $\mathrm{N}$ e $\mathrm{P}$ em solos submetidos à adubação verde com essa espécie. Assim, a aplicação de P através da adubação verde com o margaridão mostra-se vantajosa por reciclar formas indisponíveis desse nutriente no solo, tornando-o mais acessível sob a forma orgânica para as culturas de interesse (DEVIDE, 2013). Portanto, nesse estudo, os teores de $\mathrm{P}$ no solo podem ter sido influenciados pelo manejo de poda anual dessa espécie.

Os teores de $\mathrm{Ca}$ e $\mathrm{Mg}$ apresentaram uma leve redução, com exceção dos teores de Mg na linha, com elevação de $13 \%$. A redução nos teores de Ca foi de $5,4 \%$ e $17,7 \%$, na linha e entrelinha, respectivamente e o $\mathrm{Mg}$ apresentou redução na entrelinha de $16,3 \%$, em comparação com os teores desses nutrientes obtidos antes da implantação do SAF. Provavelmente tal fato se deve à quantidade desses elementos que se encontram imobilizados na vegetação. Apesar dessa leve redução nos teores desses nutrientes, que permaneceram, segundo Matiello, Garcia e Almeida (2006), em níveis elevados no solo, a saturação por bases do solo aumentou, provavelmente devido à neutralização do Al trocável e o aumento do teor de $\mathrm{K}$.

Em relação aos resultados da aplicação dos indicadores de sustentabilidade, para o parâmetro qualidade do solo foram obtidos 92 pontos no SAF e 51 pontos no café em monocultivo e para o parâmetro qualidade do café foram obtidos 34 pontos no SAF e 32 pontos no café em monocultivo

Nas Figuras 2 e 3 são apresentados gráficos do tipo radar com as notas para cada indicador avaliado, no SAF e café convencional, na Fazenda Água Limpa, em Piumhi, MG. 
Figura 2. Avaliação da qualidade do solo em cafeeiro no SAF e convencional.

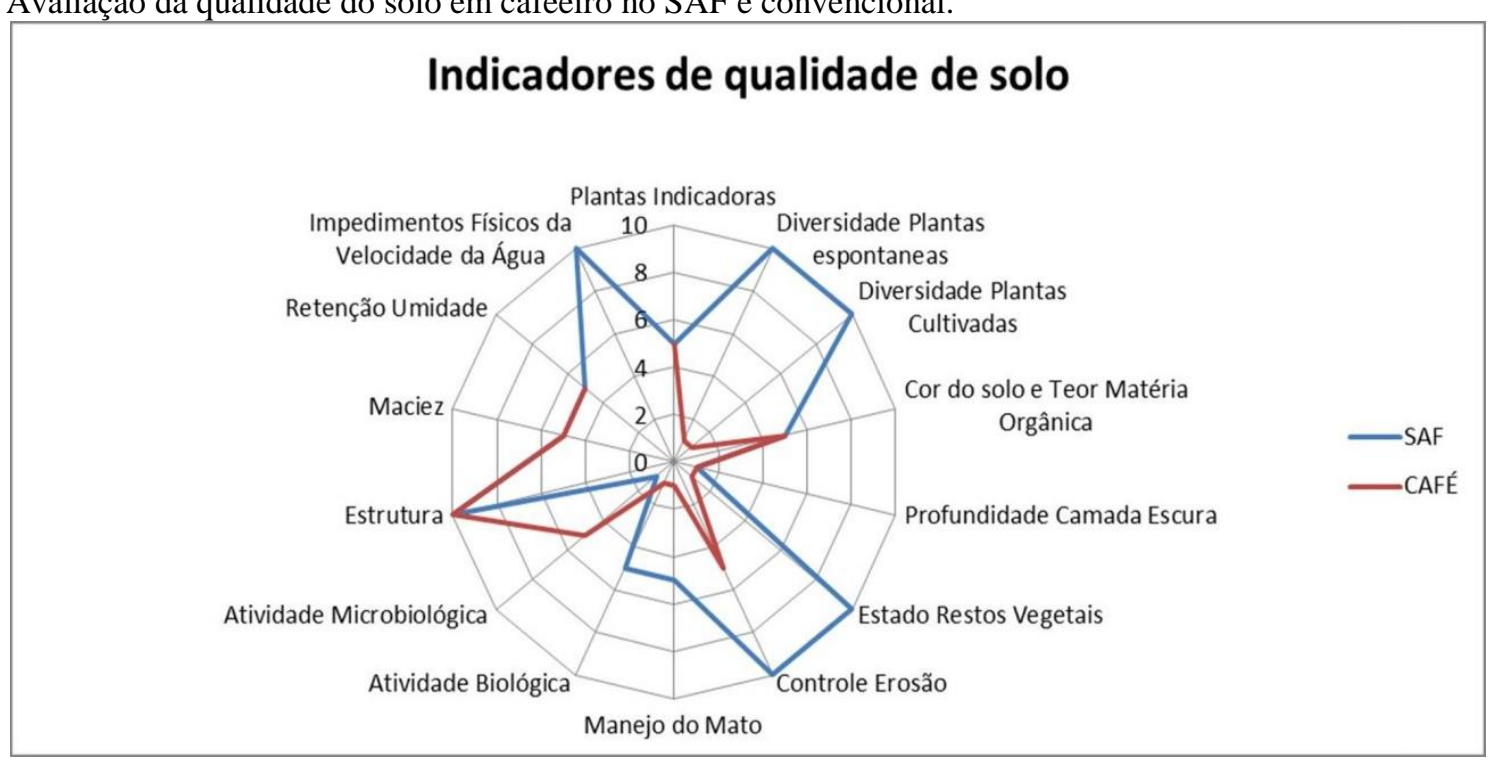

Figura 3. Avaliação da qualidade do café em SAF e convencional.

\section{Indicadores de Qualidade do Cafeeiro}
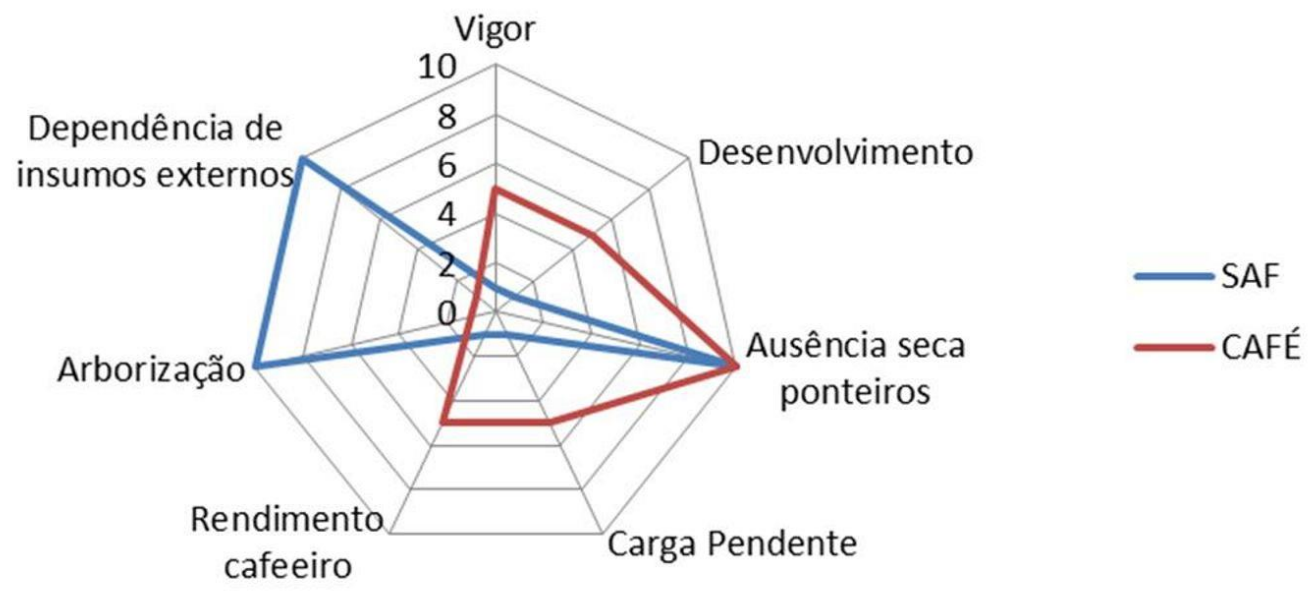

Através das Figuras 2 e 3 verifica-se que, na avaliação quanto aos indicadores de sustentabilidade, o SAF se mostrou melhor quanto aos indicadores de qualidade do solo. Entretanto, quando comparado com a lavoura convencional, os critérios agronômicos de produtividade para o SAF (vigor, desenvolvimento, carga pendente e rendimento do cafeeiro) mostraram-se baixos.

Em relação aos indicadores de qualidade do solo, apresentados na Figura 2, o SAF apresentou uma quantidade maior de indicadores com notas médias ou máximas quando comparado ao convencional. No SAF, dos 14 indicadores analisados, seis apresentaram nota máxima (10), seis obtiveram nota média (5) e apenas dois apresentaram a nota mínima. Já o convencional apresentou nota máxima (10) em apenas um indicador, com nota média (5) em sete indicadores e nota mínima (1) em seis indicadores. Grande parte dessas notas baixas relaciona-se ao fato de ser um monocultivo, com reflexos na redução da biodiversidade, promovendo uma alteração expressiva no ambiente e desequilíbrio ambiental, com consequências no empobrecimento e exaustão do solo, impactando em aspectos sociais e econômicos (ZIMMERMANN, 2009). Khatounian (2001) indica que a diversificação de culturas mostra-se fundamental para a manutenção da fertilidade dos sistemas, controle de pragas e doenças e estabilidade econômica regional. Altieri (1999) ainda aponta que a diversidade mostra-se responsável também pela regulação do microclima e processos hidrológicos, além da eliminação de organismos indesejáveis e desintoxicação de elementos químicos tóxicos.

O manejo em sistemas agroflorestais baseados em processos ecológicos, como é o caso do SAF, busca a recuperação da camada de serrapilheira sobre o solo para melhorar o fluxo de energia e ciclagem de nutrientes. Campanha et al. (2007) registraram o maior acúmulo de 
serrapilheira em café conduzido em sistema agroflorestal quando comparado com café convencional na região de Viçosa - MG, fator que contribuiu para que o sistema em SAF apresentasse maior teor médio de umidade no solo na camada de $20-40 \mathrm{~cm}$ do solo, melhorando também a saturação por bases, reduzindo o teor de alumínio trocável e aumentando a CTC do solo.

Em relação aos indicadores de qualidade do cafeeiro, apresentados na Figura 3, no SAF, as notas mais baixas foram para vigor, desenvolvimento, carga pendente e rendimento do cafeeiro, conforme pode ser observado na Tabela 5. Não há um consenso, ainda, sobre efetivas vantagens no uso de sombreamento em lavouras cafeeiras, principalmente no que diz respeito à produtividade. Moreira (2009), ao estudar o sombreamento em lavouras cafeeiras, proporcionado pela utilização da espécie Platycyamus regnellii (Pau Pereira), espécie caducifólia em dias curtos, secos e frios, em Machado (MG), obteve nas parcelas sombreadas resultados superiores de produtividade quando comparadas às parcelas com o cafeeiro cultivado a pleno sol, além de grãos de tamanhos maiores, com concentrações mais elevadas de $\mathrm{K}$ e $\mathrm{Zn}$ e melhor qualidade de bebida. Já Matiello et al. (2002) e Campanha et al. (2004), verificaram produtividades menores do cafeeiro quando em sistemas sombreados. Nesse sentido, Villatoro (2004) indica que a produtividade dos cafeeiros pode ser reduzida em sombreamento, por condições de taxas de assimilação de carbono nos ambientes reduzidos, maior estímulo ao crescimento vegetativo que ao reprodutivo e número mais baixo de nós e flores por ramo, que se mostram como componentes fundamentais na produtividade.

O manejo de podas e roçadas também pode ter influenciado os resultados para esses indicadores, uma vez que pode não ter ocorrido, pelas práticas adotadas, o fornecimento de biomassa em quantidade suficiente para o atendimento das necessidades do cafeeiro. De acordo com Matiello, Garcia e Almeida (2006) são necessários para cada saca de café a ser produzida $6,2 \mathrm{~kg}$ de $\mathrm{N}, 0,6 \mathrm{~kg}$ de $\mathrm{P}_{2} \mathrm{O}_{5}, 5,9$ $\mathrm{kg}$ de $\mathrm{K}_{2} \mathrm{O}$ e $0,3 \mathrm{~kg}$ de $\mathrm{S} \mathrm{ha}{ }^{-1}$. No SAF, a ideia inicial era a realização de roçadas de capim colonião e de plantas espontâneas nas entrelinhas trimestralmente, de modo a se ter um fornecimento mais contínuo de biomassa, associado aos resíduos de podas. Mas essa roçada trimestral ocorreu apenas no $1^{\circ}$ ano; nos anos subsequentes, a roçada foi feita uma única vez. Tal fato pode ter contribuído para nota baixa nos indicadores relacionados ao vigor e produtividade do cafeeiro no SAF de duas formas, que podem estar interligadas: baixo teor de nutrientes, devido ao baixo fornecimento de biomassa ao sistema e competição por nutrientes, água e luz com o capim colonião e plantas espontâneas localizadas nas entrelinhas que não foram manejadas adequadamente.

As notas mais elevadas para o SAF foram relacionadas à arborização, ausência de seca dos ponteiros (que também foi elevada no convencional) e dependência dos insumos externos. Um dos preceitos para a construção de sistemas agroecológicos e, por consequência, sistemas mais sustentáveis é a redução no aporte de insumos externos (insumos comerciais), utilizando recursos renováveis e disponíveis no local de produção, promovendo uma integração entre os sistemas de produção. Assim, sistemas que se mostram altamente dependentes de insumos externos, como os cafés convencionais tendem a promover problemas socioeconômicos, pelo impacto cada vez maior destes nos custos de produção agrícola e ambientais, por promoverem contaminações do ar, solo e recursos hídricos.

\section{CONCLUSÕES}

A sustentabilidade do café no sistema agroecológico avaliado mostrou-se promissora, necessitando de menor utilização de insumos externos, pois promoveu a ciclagem de nutrientes no solo com a cobertura do solo por material da roçada e pela poda das árvores, melhorando caracteres físicos, químicos e biológicos do solo. Ao mesmo tempo, a falta de cobertura do solo na região da projeção da copa do cafeeiro apresentou-se como um fator limitante no manejo do café convencional, acarretando o rápido desgaste do solo e necessidade de constantes adubações e calagens. Já de acordo com os indicadores de qualidade do café para o SAF, apenas um manejo de poda e roçada por ano no SAF não se mostraram suficientes, indicando que são necessários maiores cuidados na condução deste sistema, devendo ser feito de três a quatro roçadas ao ano do capim colonião na entrelinha e dois manejos de podas nas árvores.

\section{REFERÊNCIAS BIBLIOGRÁFICAS}

ALTIERI, M. The ecological role of biodiversity in agroecosystems. Agriculture Ecosystems \& Environment, Amsterdam, v. 74, p. 19-31, 1999.

ALTIERI, M.; NICHOLLS, C.I. Sistema agroecológico rápido de evaluácion de calidade de suelo y salud de cultivos em el agroecosistema de café. In: Un método agroecológico rápido para la evaluación de la sostentabilidad de cafetales. Manejo Integrado de Pragas y Agroecología, Costa Rica, v. 64, p. 17-24, 2002.

ARAGÃO, D.V. et al. Avaliação de indicadores de qualidade do solo sob alternativas de recuperação do solo no Nordeste paraense. Acta Amazonica, v. 42, n. 1, mar/2012. Disponível em: $<$ http://www.scielo.br/scielo.php?pid=S0044$59672012000100002 \&$ script=sci_arttext $>$. Acesso em 30 nov. 2014.

BAYER, C.; MIELNICZUCK, J. Dinâmica e função da matéria orgânica. In: SANTOS, G. de A. et al. (ed.). Fundamentos da matéria orgânica do solo. Porto Alegre: Gráfica Metrópole, 2008, p. 8-17.

BEER, J.; MUSCHLER, R.; KASS, D; SOMARRIBA, E. Shade management in: coffee and cacao plantations. Agroflorestry Systems, v. 38, 1998. p. $139-164$.

CAMPANHA, M.M.; SANTOS, R.H.S.; FREITAS, G.B.; MARTINEZ, H.E.P.; GARCIA, S.L.R.; FINGER, F.L. Growth and yield of coffee plants in agroforestry and monoculture systems in Minas Gerais, Brazil. Agroforestry Systems, Dordrecht, v. 63, p. 75-82, 2004.

CAMPANHA, M. M.; SANTOS, R. H. S.; FREITAS, G. B.; MARTINEZ, H. E. P.; BOTERO, C. J.; GARCIA, S. L. Análise Comparativa das Características da Serrapilheira e Do Solo Em Cafezais (Coffea arábica L.) Cultivados em 
Sistema Agroflorestal e em Monocultura, na Zona da Mata MG. Revista Árvore, Viçosa, v. 31, n. 5, p. 805-812, 2007.

CARVALHO, L.C.T. Análise comparativa entre diferentes metodologias utilizadas para recomendação de gesso agrícola em solos cultivados com lavoura de café na região sul de Minas Gerais. 2008. 37f. Trabalho de Conclusão de Curso (Graduação em Cafeicultura) - Escola Agrotécnica Federal de Muzambinho, Muzambinho, 2008.

CORREIA, M. E. F.; ANDRADE, A. G. Formação de Serrapilheira e Ciclagem de Nutrientes. In: SANTOS, G. A.; CAMARGO, F. A. O.; (Ed.). Fundamentos da Matéria Orgânica do Solo. Ecossistemas Tropicais. Porto Alegre: Editora Gênesis, 1999. p. 197-223.

DEPONTI, C. M.; ECKERT, C.; AZAMBUJA, J. L. B. Estratégia para construção de indicadores para avaliação da sustentabilidade e monitoramento de sistemas. Agroecologia e Desenvolvimento Rural Sustentável, v. 3, n. 4, p. 44-52, out./dez. 2002. Disponível em: <http://www.ernestoamaral.com/docs/indsoc122/biblio/Deponti2002.pdf>. Acesso em: 15 out. 2014.

DEVIDE, A.C.P. Adubos verdes para sistemas agroflorestais com guanandi cultivado em várzea e terraço fluvial. 2013, 36f. Qualificação (Doutorado em Fitotecnia) Universidade Federal Rural do Rio de Janeiro, 2013. Disponível

em: <http://orgprints.org/24817/1/ADUBACAO_VERDE_CA LOPHYLLUM_SAF.pdf>. Acesso em 01 dez. 2014.

FREITAS, R. B.; OLIVEIRA, L. E. M.; FILHO, N. D.; SOARES, A. M. Influência de diferentes níveis de sombreamento no comportamento fisiológico de cultivares de café (Coffea arabica L). Ciência e Agrotécnica, Lavras, v. 27, n. 4, p. 804-810, 2003

GUIMARÃES, P. T. G.; GARCIA, A. W. R.; ALVAREZ VIEGAS, V. H.; PREZOTTI, L. C.; VIANA, A. S.; MIGUEL, A. E.; MALAVOLTA, E.; CORRÊA, J. B.; LOPES, A. S.; NOGUEIRA, F. D.; MONTEIRO, A. V. C. Cafeeiro. In: RIBEIRO, A. C.; GUIMARÃES, P. T. G.; ALVAREZ VIEGAS, V. H. (Ed.). Recomendações para o uso de corretivos e fertilizantes em Minas Gerais: $5^{\mathrm{a}}$ aproximação. Viçosa, MG: Comissão de Fertilidade do Solo do Estado de Minas Gerais, 1999. p. 289-302.

GLIESSMAN, S. R. Agroecologia: Processos Ecológicos em Agricultura Sustentável. Porto Alegre/RS. UFRGS Editora, 2005. p. 475-507.

KHATOUNIAN, C. A. A reconstrução ecológica da agricultura. Botucatu: Agroecológica, 2001.

KIEHL, E. J. Adubação Orgânica. 500 Perguntas \& Respostas. Piracicaba: Editora Degaspari, 2008. p. 11

KUMAR, D.; TIESZEN, L. L. Photosyntesis in Coffea arabica. I. Effects of light and temperature. Experimental Agriculture, Trinidad, v. 16, n. 1, p. 13-16, 1980.
KÖPPEN, W. Climatologia: con um estúdio de los climas de la tierra. México: Fondo de Cultura Economica, 1948. 478 p.

MATIELLO, J.B.; SANTINATO, R.; GARCIA, A.W.R.; ALMEIDA, S.R.; FERNANDES, D.R. Cultura de café no Brasil: novo manual de recomendações. Rio de Janeiro: MAPA/PROCAFE, 2002, 387 p.

MATIELLO, J.B.; GARCIA, A.W.R.; ALMEIDA, S.R Adubos, corretivos e defensivos para a lavoura cafeeira. Varginha: MAPA/Fundação PROCAFÉ, 2006. 89 p.

MOREIRA, C.F. Sustentabilidade de produção de sistemas de café sombreado orgânico e convencional. 2009. $145 f$. Tese (Doutorado em Ecologia Aplicada) - Escola Superior de Agricultura "Luiz de Queiroz", Universidade de São Paulo, Piracicaba, 2009.

MOURA, L.G.V.; ALMEIDA, J.; MIGUEL, L.A. Avaliação de sustentabilidade em agroecossistemas: um pouco de pragmatismo. REDES, Universidade de Santa Cruz do Sul (UNISC), v. 9, n. 2 (maio/ agosto de 2004) - Santa Cruz do Sul: Editora da UNISC, 2004. p. 133-155.

NG'INJA, J.O. et al. Traditional hedges in western Kenya: typology, composition, distribution, uses, productivity and tenure. Pilot Project No. 8. Regional Agroforestry Research Centre, Maseno, Kenya. 1998.

NUNES, M.A.; RAMALHO, J.D.C.; DIAS, M.A. Effect of nitrogen supply on the photosynthetic performance of leaves from coffee plants exposed to bright light. Journal of Experimental Botany, London, v. 44, n. 4, p. 893-899, 1993.

PAVINATO, P.S.; ROSOLEM, C.A. Disponibilidade de nutrientes no solo: Decomposição e Liberação de Compostos Orgânicos e Resíduos Vegetais. Revista Brasileira De Ciência do Solo, v. 32, n. 3, 2008. p. 911-920. Disponível em: <http://www.scielo.br/scielo.php?pid=S0100$06832008000300001 \&$ script $=$ sci_arttext $>$. Acesso em 10 out. 2014

PEREZ MARIN, A. M. Impactos de um sistema agroflorestal com café na qualidade do solo. 2002. 83 p. Dissertação (Mestrado). Universidade Federal de Viçosa, Viçosa, MG.

PRIMAVESI, A. Manejo ecológico do solo: a agricultura em regiões tropicais. São Paulo: Nobel, 2002. 549 p.

PYPERS, P.; VERSTRAETE, S.; THI, C.P.; MERCKX, R. Changes in mineral nitrogen, phosphorus availability and salt-extractable aluminium following the application of green manure residues in two weathered soils on South Vietnan. Soil Biology and Biochemistry, Oxford, v.37, n.1, p.163-172, jan. 2005 .

RAIJ, B. van; CANTARELLA, H.; QUAGGIO, J.A.; FURLANI, A.M.C, Recomendações de adubação e calagem para o Estado de São Paulo. Campinas: IAC, 1997. 185 p. 
RAMALHO, J. D. C.; CAMPOS, P. S.; TEXEIRA, M.; NUNES, M. A. Nitrogen dependent changes in antioxidant system and in fatty acid composition of chloroplast membranes from Coffea arabica L. submitted to high irradiance. Plant Science, Himerick, v. 135, n. 2, 1998, p. 115-124.

ROSCOE, R.; BODDEY, R. M.; SALTON, J. C. Sistemas de Manejo e Matéria Orgânica no Solo. In: ROSCOE, R.; MERCANTE, F. M.; SALTON, J. C. Dinâmica da Matéria Orgânica do Solo em Sistemas Conservacionistas: Modelagem Matemática e Métodos Auxiliares. DouradosMS. Embrapa, 2006. p. 17-42.

SOMMARIBA, E., Revisiting the past: an essay on agroforestry definition. Agroforestry Systems, n. 19, p. 233-240, 1992.

SOUZA JÚNIOR, O.F. de. Influência do espaçamento e da época de corte na produção de biomassa e valor nutricional de Tithonia diversifolia (HEMSL.) Gray. 2007, 43f. Dissertação (Mestrado em Agronomia) - Faculdade de Ciências Agrárias, Universidade de Marília, Marília, $2007 . \quad$ Disponível em: $<$ http://www.unimar.br/pos/trabalhos/arquivos/16FBB429 41C786FA4ACAB44672B0A3F9.pdf>. Acesso em 30 nov. 2014.

VERONA, L. A. F; CASALINHO, H.; MASERA, O.; GALVÁN, Y.; CORRÊA, I.; SCHWENGBER, J. Uso de indicadores compostos na análise da sustentabilidade de agroecossistemas de base familiar na região sul do Rio Grande do Sul. Revista Brasileira de Agroecologia, v. 2, n. 2, 2007. Disponível em: <http://www.abaagroecologia.org.br/revistas/index.php/rbagroecologia/arti cle/view/7191/5277>. Acesso em: 15 out. 2014.

VILLATORO, M.A.A. Matéria Orgânica e Indicadores Biológicos da Qualidade do Solo na Cultura do Café sob Manejo Agroflorestal e Orgânico. 2004. 166f. Tese (Doutorado em Ciências em Agronomia) - Universidade Federal Rural do Rio de Janeiro, Seropédica, 2004.

ZIMMERMANN, C.L. Monocultura e transgenia: impactos ambientais e insegurança alimentar. Veredas do Direito, Belo Horizonte, v. 6, n. 12, p. 79-100, jul/dez 2009. Disponível em: <http://www.domhelder.edu.br/revista/index.php/veredas/ article/view/21>. Acesso em: 07 nov. 2014. 